\title{
Consumo de álcool no padrão binge e suas consequências em usuários de drogas
}

\section{em tratamento}

Maria Fernanda Rosa de Almeida Raimundo ${ }^{1}$, Natália Priolli Jora Pegoraro ${ }^{2}$, Josélia Benedita Carneiro Domingos $^{3}$, Angélica Martins de Souza Gonçalves ${ }^{4}$, Jessica Adrielle Teixeira Santos ${ }^{5}$, Sandra Cristina Pillon ${ }^{6}$

\footnotetext{
${ }^{1}$ Enfermeira, Mestre em Enfermagem Psiquiátrica. Ribeirão Preto, SP, Brasil. E-mail: maria.raimundo@usp.br.

${ }^{2}$ Enfermeira, Doutora em Enfermagem Psiquiátrica. Enfermeira Coordenadora do Centro de Atenção Psicossocial de Ribeirão Preto. Ribeirão Preto, SP, Brasil. E-mail: natalia.jora@usp.br.

${ }^{3}$ Enfermeira, Doutora em Enfermagem Psiquiátrica. Enfermeira Coordenadora de Unidade Básica de Saúde de Jaboticabal. Jaboticabal, SP, Brasil. E-mail: joselia.rocci@hotmail.com.

${ }^{4}$ Enfermeira, Doutora em Enfermagem Psiquiátrica. Professora Adjunto da Universidade Federal de São Carlos. São Carlos, SP, E-mail: angelicamartins@ufscar.br.

${ }^{5}$ Enfermeira, Mestre em Enfermagem. Discente do Programa de Pós-Graduação em Enfermagem Psiquiátrica, nível Doutorado, da Escola de Enfermagem de Ribeirão Preto da Universidade de São Paulo (EERP/USP). Ribeirão Preto, SP, Brasil. E-mail: jessicadrielle@yahoo.com.br.

${ }^{6}$ Enfermeira, Doutora em Psiquiatria e Psicologia Médica. Professor Titular EERP/USP. Ribeirão Preto, SP, Brasil. E-mail: pillon@eerp.usp.br.
}

Recebido: 24/07/2015

Aceito: 03/12/2015

Publicado: 30/06/2016.

Como citar esse artigo:

Raimundo MFRA, Pegoraro NPJ, Domingos JBC, Gonçalves AMS, Santos JAT, Pillon SC. Consumo de álcool no padrão binge e suas consequências em usuários de drogas em tratamento. Rev. Eletr. Enf. [Internet]. 2016 [acesso em:_____18:e1158. Disponível em: http://dx.doi.org/10.5216/ree.v18.36833.

\section{RESUMO}

O objetivo foi avaliar o uso de álcool no padrão binge e suas consequências em usuários de drogas. Estudo descritivo, de abordagem quantitativa, desenvolvido com 140 indivíduos de um Centro de Atenção Psicossocial - Álcool e Drogas. Foram aplicados: um formulário de informações sociodemográficas, a escala de gravidade de dependência de álcool e escala de severidade do uso de drogas. A amostra caracterizou-se por serem adultos do sexo masculino $(76,4 \%)$, solteiros (77,1\%), de cor negra/parda, com baixo nível de escolaridade $(57,1 \%)$ e religião católica $(55,8 \%)$. Eram usuários de crack $(38,6 \%)$, de múltiplas drogas $(32,1 \%)$ e de cocaína $(29,3 \%)$, a maioria $(80,7 \%)$ fez uso de álcool no padrão binge e apresentou níveis severos de dependência alcoólica (93,8\%). Ameaça, agressividade e brigas foram evidenciadas na amostra. Os resultados indicam a necessidade de oferecer estratégias interventivas mais intensivas para controle do uso de álcool nesse segmento vulnerável da população, visando minimizar danos.

Descritores: Transtornos Relacionados ao Uso de Álcool; Bebedeira; Cuidados de Enfermagem; Cocaína; Cocaína Crack.

\section{INTRODUÇÃO}

Segundo a Organização Mundial de Saúde (OMS), as consequências do uso nocivo do álcool resultam, anualmente, em cerca de 2,5 milhões de mortes $^{(1)}$. O uso nocivo do álcool é responsável pela morte de, 
aproximadamente, 320.000 jovens em fase produtiva, o que supera o tabagismo como fator de risco para diversas doenças e também representa risco seis vezes maior do que o uso de drogas ilícitas. 0 álcool possui propriedades que causam dependência e afetam, de forma heterogênea, a população, com suas peculiaridades de vulnerabilidade em cada segmento social. As consequências de seu uso nocivo são evidentes nas esferas social e econômica e no sistema de saúde, devido aos altos índices de morbimortalidade que levam o indivíduo à incapacidade ${ }^{(1)}$.

Os resultados do levantamento nacional sobre os padrões de consumo de álcool na população brasileira evidenciaram que metade dos brasileiros acima de 18 anos bebeu pelo menos uma vez ao ano, sendo a maioria do sexo masculino. Nessa amostra, $11 \%$ dos entrevistados relataram beber todos os dias e $28 \%$ entre uma e quatro vezes na semana. Identificou-se ainda que $38 \%$ dos homens que beberam no último ano consumiram bebidas alcoólicas no padrão binge ${ }^{(2)}$.

Atualmente, as pesquisas vêm destacando um determinado tipo de ingestão, cada vez mais frequente e com importantes consequências deletérias à saúde: o binge drinking. Trata-se de um padrão caracterizado pelo consumo de grande quantidade de álcool em um período curto de tempo, prática descrita na literatura como binge drinking, ou beber em binge. Esse termo é empregado para definir o "uso pesado episódico do álcool". Caracteriza um tipo de consumo de risco elevado e frequentemente associado a uma série de problemas físicos, sociais e mentais ${ }^{(1-4)}$. Esse padrão resulta em importantes modificações neurofisiológicas (desinibição comportamental, comprometimento cognitivo, diminuição da atenção, piora da capacidade de julgamento e diminuição da coordenação motora) $)^{(4)}$.

As características do consumo de álcool e suas complicações estão relacionadas ao volume de bebida ingerido e à frequência de uso ${ }^{(1-6)}$. Os bebedores que consomem no padrão binge apresentam maiores danos quando comparados a indivíduos que consomem em um padrão contínuo, porém em menor volume. 0 consumo em binge é descrito como um comportamento que potencializa os danos associados ao uso do álcool, representando risco para a saúde e elevados custos econômicos e sociais ${ }^{(5)}$.

O beber em binge está presente em diferentes culturas, cada qual com suas especificidades, sendo identificado predominantemente na população jovem e adulta. $\mathrm{O}$ sexo masculino tende a consumir mais quando comparado ao feminino ${ }^{(6)}$.

Estudo constatou que o consumo de álcool no padrão binge vem aumentando nos Estados Unidos da América, sendo que $17,1 \%$ da população consumiram nesse padrão com maior frequência e intensidade. Nesse estudo, os indivíduos que iniciaram o consumo em idade precoce de álcool apresentaram três vezes mais chances de consumir no padrão binge, porém foi observado que os episódios de beber pesado tendem a diminuir com a idade ${ }^{(7)}$.

Quando associado a outras drogas, o álcool é um importante fator causal para o desenvolvimento de mais de 60 tipos de doenças. Segundo a Organização Mundial da Saúde $(\mathrm{OMS})^{(1)}$, o uso nocivo está relacionado aos principais fatores que contribuem para o início de transtornos neuropsiquiátricos, epilepsia e outras condições crônicas não transmissíveis, doenças cardiovasculares e cirrose hepática. O uso da 
substância também está relacionado a comportamentos sexuais que expõem o indivíduo a riscos, como a infecção por HIV/Aids e outras doenças sexualmente transmissíveis. $\mathrm{O}$ uso nocivo de álcool também pode contribuir para que o indivíduo mantenha múltiplos parceiros sexuais e se engaje em relações sexuais desprotegidas $^{(1,8)}$.

O consumo de álcool associado à cocaína é uma das combinações mais frequentes, que vem se tornando um hábito cada vez mais comum entre os usuários de drogas. Essa associação acarreta problemas de saúde pública, pois potencializa uma série de complicações clínicas, psiquiátricas e sociais, contribuindo para o incremento dos índices de morbimortalidade. Observa-se, na prática clínica e em pesquisas, que os dependentes de cocaína e crack, em geral, são também dependentes de outras substâncias psicoativas e fazem uso problemático de álcool ${ }^{(9)}$.

No organismo, a interação entre essas duas drogas produz uma substância denominada cocaetileno, que apresenta mecanismos de ação tóxica semelhante e mais elevada do que a própria cocaína ${ }^{(10)}$. Esse metabólito da cocaína é formado na presença do etanol, de modo que sua lenta remoção pelo organismo torna a associação um importante atrativo. Estudos apontam que o uso crônico de álcool e/ou de cocaína/crack pode desencadear o desenvolvimento de quadros psiquiátricos, principalmente transtornos do humor ${ }^{(11)}$, bem como originar sérios problemas cardiovasculares e de hepatotoxidade, perda crescente do controle do consumo, aumento prolongado da euforia, problemas sociais e condutas violentas, que levam à exposição a comportamentos de alto risco, constituindo a base para quadros clínicos de maior gravidade ${ }^{(12)}$.

Estudos identificam forte relação entre o uso frequente de álcool e cocaína. Essas substâncias psicoativas provocam elevados níveis de ansiedade e desejo, que tornam o indivíduo mais suscetível a recaídas durante o tratamento, além de aumentar sua vulnerabilidade ao estresse e ao uso de outras drogas $^{(13)}$. Indivíduos usuários de múltiplas drogas apresentam elevado comprometimento de suas funções cognitivas, como memória e raciocínio lógico, e funções executivas, como planejamento, execução de diversas tarefas, fluência verbal, entre outros ${ }^{(4,8-9,11)}$.

Estudos que avaliaram o uso de álcool no padrão binge em usuários de cocaína, nas suas diferentes formas (inalada e fumada), que se encontram em tratamento, ainda são escassos no Brasil. A maioria das pesquisas são de natureza descritiva e de levantamentos. Os disponíveis mostraram apenas avaliações das consequências para cada droga de forma separada. $\mathrm{O}$ uso de álcool em binge e suas consequências vão além da natureza clínica, pois perpassam os problemas familiares, sociais e culturais, que muitas vezes são indissociáveis, mas que podem ser prevenidos precocemente. Desse modo, torna-se de extrema importância conhecer o perfil dessa população, que vem crescendo nos programas assistenciais, assim, identificar suas especificidades e compreender as características relacionadas ao beber em binge, a fim de planejar uma assistência de qualidade a essas pessoas.

No cenário nacional um dos desafios encontrados no contexto das práticas clínicas dos profissionais de serviços substitutivos de saúde mental é implementar estratégias de controle para minimizar as consequências nocivas desse padrão de consumo, uma vez que a maioria dos usuários apresenta quadros 
crônicos de uso do álcool. Nesse sentido, o presente estudo explora algumas lacunas e possibilidades concretas

transformação dessas práticas no âmbito da prevenção de agravos maiores.

O presente estudo teve por objetivo avaliar o uso de álcool no padrão binge e suas consequências em usuário de drogas em tratamento para a dependência química.

\section{MATERIAIS E MÉTODOS}

Trata-se de um estudo transversal, exploratório, de abordagem quantitativa.

A pesquisa foi desenvolvida no Centro de Atenção Psicossocial para Usuários de Álcool e outras Drogas (CAPSad), localizado em município de médio porte do interior do Estado de São Paulo.

A amostra foi composta por 140 usuários de drogas que procuraram tratamento pela primeira vez no referido serviço. O recrutamento ocorreu por meio da constituição de uma amostra consecutiva de indivíduos que buscaram atendimento por demanda espontânea ou encaminhamento da rede.

Os critérios de elegibilidade foram: ser clientes de ambos os sexos, com idade igual ou superior a 18 anos, cadastrados no referido serviço. Foram critérios de exclusão: indivíduos que apresentaram retardo mental grave, sintomas psicóticos evidentes e prejuízos cognitivos severos, avaliados por meio da Escala Breve de Avaliação Psiquiátrica. Nenhum usuário convidado recusou-se a participar do estudo ou foi eliminado com base na aplicação dos critérios de exclusão.

Os participantes foram recrutados nas atividades terapêuticas de rotina do referido serviço, ou seja, no primeiro atendimento individual ou após a participação no grupo de acolhimento. Essas atividades foram escolhidas considerando que todos os usuários admitidos passam por consulta com a enfermeira ou com outro profissional da equipe de saúde. Os participantes foram apresentados aos pesquisadores pela enfermeira do serviço e esclarecidos quanto aos procedimentos da pesquisa. Mediante a aceitação, assinaram o Termo de Consentimento Livre e Esclarecido, sendo-Ihes assegurado o recebimento de uma via. A entrevista foi agendada em local privativo. A coleta de dados foi realizada no período de fevereiro a junho de 2012.

Realizou-se um treinamento prévio com os pesquisadores, a fim de padronizar os procedimentos de coleta de dados. Para a coleta de dados elaborou-se um questionário contendo: (1) Informações sociodemográficas; (2) Escala de Gravidade de Dependência do Álcool (SADD); (3) Teste de Identificação do Uso de Álcool - C (AUDIT-C); (4) Escala de Severidade da Dependência de Drogas (SDS).

(1) Formulário de informações sociodemográficas: composto por idade, sexo, cor, estado civil, escolaridade e religião.

(2) O Short Alcohol Dependence Data (SADD) foi traduzido e validado para o português do Brasil ${ }^{(14)}$. Trata-se de uma escala constituída por 15 itens relacionados à gravidade da dependência do álcool. De acordo com o somatório total de pontos, os usuários são classificados como dependência: leve (1 a 9), moderada (10 a 19) e grave (20 a 45). 
(3) Teste de Identificação do Uso de Álcool - C (AUDIT-C): trata-se de uma versão abreviada do AUDIT. Esse teste contém três itens referentes à quantidade, frequência e consumo no padrão binge. Para a leitura dos níveis de risco de consumo é necessário proceder ao somatório das respostas, que variam de zero a 12 pontos, sendo diferenciada a pontuação entre os sexos: escores a partir de quatro pontos para o feminino e cinco para o masculino, o que indica um consumo nocivo e direciona a intervenção ${ }^{(15)}$. No presente estudo foi utilizado apenas o terceiro item desse instrumento, referente à avaliação do uso abusivo de álcool no padrão binge.

(4) Escala de Severidade da Dependência de Drogas (SDS): mensura o nível de gravidade da dependência de cocaína e/ou crack $^{(10,16)}$. Cada um dos cinco itens possui um escore de respostas de quatro pontos. A pontuação total é obtida por meio da soma dos cinco itens, que mensuram os sintomas da síndrome de dependência nos últimos 12 meses. Para a leitura dos resultados, quanto maior a pontuação obtida, maior o nível de gravidade da dependência da droga ${ }^{(10,16)}$.

O projeto foi submetido e aprovado pelo Comitê de Ética em Pesquisa do Centro Saúde Escola da Faculdade de Medicina de Ribeirão Preto da Universidade de São Paulo, Processo $n^{\circ} 381$, de acordo com o preconizado pela Resolução 466/2012.

Para análise do material, elaborou-se um banco de dados no Statistical Program of Social Science (SPSS) versão. 19, for Windows. Foi realizada a análise exploratória por meio de médias, frequência e porcentagem dos dados, para elucidação das características da amostra. Utilizou-se o Teste Exato de Fisher e o teste Qui-quadrado para mensurar o grau de associação entre duas variáveis, para testar a significância existente entre duas variáveis qualitativas, comparar proporções e as possíveis divergências entre as frequências observadas e esperadas para um certo evento. Foi considerado o nível do nível de significância $p<0,05$, Intervalo de Confiança (IC) $95 \%$.

\section{RESULTADOS}

A amostra foi composta por $140(100 \%)$ usuários de drogas, dos quais $113(80,7 \%)$ consumiram no padrão binge. Os participantes foram predominantemente do sexo masculino, adultos, solteiros, de cor negra/parda, com baixo nível de escolaridade e que professavam a religião católica. Os pacientes que consumiram ou não bebidas alcoólicas no padrão binge não se diferenciaram em relação às características sociodemográficas na presente amostra (Tabela 1).

Quanto ao uso de drogas, 38,6\% dos participantes eram usuários de crack, 32,1\% de múltiplas drogas e $29,3 \%$ de cocaína.

A frequência do consumo de álcool no padrão binge foi maior em participantes que consumiram de um a dois dias na semana e diariamente, com associações estatisticamente significantes quando comparados aos que não consumiam nesse padrão (Tabela 2).

O consumo de álcool no padrão binge ocorreu em maior porcentagem em usuários de múltiplas drogas, com frequência de consumo regular nos últimos 30 dias e diariamente, quando comparados aos 
usuários de cocaína e de crack, com diferenças estatisticamente significantes (Tabela 3).

Tabela 1: Informações sociodemográficas e binge-drinking nos usuários de drogas ( $N=140$ ). Ribeirão Preto, SP, Brasil, 2015.

\begin{tabular}{|c|c|c|c|c|c|c|c|c|}
\hline & & \multirow{2}{*}{\multicolumn{2}{|c|}{ Total }} & \multicolumn{4}{|c|}{ BD } & \multirow{3}{*}{ Valor de $p$} \\
\hline & & & & & & & co & \\
\hline & & $n$ & $\%$ & $n$ & $\%$ & $\mathbf{n}$ & $\%$ & \\
\hline \multicolumn{2}{|r|}{ Total } & 140 & 100,0 & 113 & 80,7 & 27 & 19,3 & \\
\hline \multirow{3}{*}{ Sexo } & Masculino & 107 & 76,4 & 86 & 80,4 & 21 & 19,6 & \multirow{2}{*}{$0,538^{\mathrm{a}}$} \\
\hline & Feminino & 33 & 23,6 & 27 & 81,8 & 16 & 18,2 & \\
\hline & 18 a 29 anos & 51 & 36,4 & 38 & 74,5 & 13 & 25,5 & \multirow{3}{*}{$0,128^{b}$} \\
\hline \multirow[t]{2}{*}{ Faixa etária } & 30 a 49 anos & 68 & 48,6 & 55 & 80,9 & 13 & 19,1 & \\
\hline & 50 anos ou mais & 21 & 15,0 & 20 & 95,2 & 1 & 4,8 & \\
\hline \multirow{2}{*}{ Raça } & Negra/Preta & 76 & 54,3 & 61 & 80,3 & 15 & 19,7 & \multirow{2}{*}{$0,528^{a}$} \\
\hline & Branca & 64 & 45,7 & 52 & 81,3 & 12 & 18,8 & \\
\hline \multirow{2}{*}{ Estado civil } & União consensual & 32 & 22,9 & 27 & 84,4 & 5 & 15,6 & \multirow{2}{*}{$0,376^{a}$} \\
\hline & Sozinho & 108 & 77,1 & 86 & 79,6 & 22 & 20,4 & \\
\hline \multirow{3}{*}{ Escolaridade } & Ensino Fundamental & 80 & 57,1 & 70 & 87,5 & 10 & 12,5 & \multirow{3}{*}{$0,061^{b}$} \\
\hline & Ensino Médio & 49 & 35,0 & 37 & 71,2 & 15 & 28,8 & \\
\hline & Ensino Superior & 8 & 5,9 & 6 & 75,0 & 2 & 25,0 & \\
\hline \multirow{3}{*}{ Religião } & Católico & 58 & 55,8 & 47 & 81,0 & 11 & 19,0 & \multirow{3}{*}{$0,359^{b}$} \\
\hline & Evangélico & 37 & 35,6 & 31 & 83,8 & 6 & 16,2 & \\
\hline & Não tem & 9 & 8,6 & 9 & 100,0 & - & - & \\
\hline
\end{tabular}

${ }^{\mathrm{a}}$ Teste Exato de Fisher. ${ }^{\mathrm{b}}$ Teste Qui-quadrado. ${ }^{(*)} \mathrm{p} \geq 0,05$

Tabela 2: Frequência do binge-drinking, nos últimos seis meses, segundo os usuários de drogas ( $N$ = 140). Ribeirão Preto, SP, Brasil, 2015.

\begin{tabular}{|c|c|c|c|c|c|c|}
\hline & & \multicolumn{4}{|c|}{ BD } & \multirow{3}{*}{ Valor de $p$} \\
\hline & & \multicolumn{2}{|c|}{ Não } & \multicolumn{2}{|c|}{ Sim } & \\
\hline & & $\mathbf{N}$ & $\%$ & $\mathbf{N}$ & $\%$ & \\
\hline \multirow{5}{*}{ Frequência } & Sem uso & 25 & 2,5 & 15 & 37,5 & \multirow{5}{*}{$0,000 *$} \\
\hline & 1-3 vezes por mês & 1 & 10,0 & 9 & 90,0 & \\
\hline & 1-2 vezes por semana & - & - & 11 & 100,0 & \\
\hline & 3-6 vezes por semana & 1 & 4,0 & 24 & 96,0 & \\
\hline & Diariamente & - & - & 54 & 100,0 & \\
\hline
\end{tabular}

Teste Qui-quadrado. ${ }^{(*)} \mathrm{p} \leq 0,05$

Tabela 3: Tipo de droga e frequência do binge-drinking, segundo os usuários de drogas ( $N$ 140). Ribeirão Preto, SP, Brasil, 2015.

\begin{tabular}{|c|c|c|c|c|c|c|c|c|}
\hline & & \multicolumn{6}{|c|}{ Drogas usadas } & \multirow{3}{*}{ Valores de $p$} \\
\hline & & \multicolumn{2}{|c|}{ Cocaína } & \multicolumn{2}{|c|}{ Crack } & \multicolumn{2}{|c|}{ Múltiplas drogas } & \\
\hline & & $\mathbf{N}$ & $\%$ & $\mathbf{N}$ & $\%$ & $\mathbf{N}$ & $\%$ & \\
\hline \multirow{2}{*}{ 1. Consumo regular (três ou mais dias/semana) } & Não & 13 & 35,1 & 24 & 64,9 & - & - & \multirow{2}{*}{$0,000 *$} \\
\hline & Sim & 28 & 27,2 & 30 & 29,1 & 45 & 43,7 & \\
\hline \multirow{2}{*}{ 2. Consumo nos últimos 30 dias } & Não & 24 & 38,7 & 28 & 45,2 & 10 & 16,1 & \multirow{2}{*}{$0,001^{*}$} \\
\hline & Sim & 17 & 22,1 & 25 & 32,5 & 35 & 45,5 & \\
\hline \multirow{2}{*}{ 3. Consumo diariamente nos últimos $\mathbf{5 0}$ dias ou mais } & Não & 10 & 37 & 17 & 63 & - & - & \multirow{2}{*}{$0,000 *$} \\
\hline & Sim & 31 & 27,4 & 37 & 32,7 & 45 & 39,8 & \\
\hline
\end{tabular}
Teste Exato de Fisher. ${ }^{(*)} p \leq 0,05$

Os usuários que consumiram no padrão binge apresentaram em maior porcentagem nível de gravidade de dependência de álcool (SADD) severo (Sim 93,8\% versus 6,2\% Não), moderado (Sim 97,1\% Rev. Eletr. Enf. [Internet]. 2016 [acesso em:______];18:e1158. Disponível em: http://dx.doi.org/10.5216/ree.v18.36833. 
versus 2,9\% Não), leve (Sim 82,6\% versus 17,6\% Não) e sem dependência do álcool (Sim 17,4\% versus 82,6\%), com diferenças estatisticamente significantes $(p \leq 0,05)$.

Ao comparar as escalas de dependência de drogas (SDS) e de álcool (SADD) e o binge, os usuários que consumiram nesse padrão apresentaram valores médios maiores $(20,7 \pm 10,3 t=6,3 \mathrm{IC} 19,0-9,9)$ apenas nos níveis de gravidade de dependência do álcool (SADD), quando comparados aos que não consumiam nesse padrão $(6,2 \pm, 3,5 t=5,6$ IC 19,7 - 9,3), com valores estatisticamente significantes ( $p>0,05)$. Em relação a

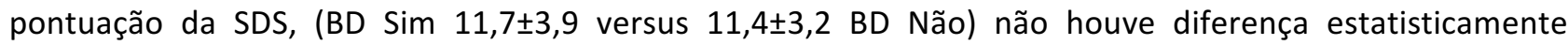
significante $(p>0,05)$.

Embora a maioria das situações de violência tenha predominado entre participantes que consumiam álcool no padrão binge, não houve diferenças estatisticamente significantes quando comparados aos que não consumiam nesse padrão (Tabela 4).

Tabela 4: Frequência de situações de violência após uso de cocaína/crack e binge-drinking, segundo os usuários de drogas ( $N$ = 140). Ribeirão Preto, SP, Brasil, 2015.

\begin{tabular}{|c|c|c|c|c|c|c|}
\hline & & \multicolumn{4}{|c|}{ BD } & \multirow{3}{*}{ Valor de $p$. } \\
\hline & & \multicolumn{2}{|c|}{ Não } & \multicolumn{2}{|c|}{ Sim } & \\
\hline & & $\mathbf{N}$ & $\%$ & $\mathbf{N}$ & $\%$ & \\
\hline \multirow{2}{*}{ Ameaçado por violência física } & Não & 14 & 15,4 & 77 & 84,6 & \multirow{2}{*}{0,085} \\
\hline & Sim & 13 & 26,5 & 36 & 73,5 & \\
\hline \multirow{2}{*}{ Agressividade } & Não & 9 & 24,3 & 28 & 75,7 & \multirow{2}{*}{0,064} \\
\hline & Sim & 18 & 22,2 & 63 & 77,8 & \\
\hline \multirow{2}{*}{ Envolvimento em brigas } & Não & 16 & 25,8 & 46 & 74,2 & \multirow{2}{*}{0,283} \\
\hline & Sim & 11 & 19,6 & 45 & 80,4 & \\
\hline \multirow{2}{*}{ Encorajado a lutar por si mesmo } & Não & 18 & 27,3 & 48 & 72,7 & \multirow{2}{*}{0,145} \\
\hline & Sim & 9 & 17,3 & 43 & 82,7 & \\
\hline
\end{tabular}

Teste Exato de Fisher. Valores de $\mathrm{p} \leq 0.05$

\section{DISCUSSÃO}

O presente estudo avaliou o consumo de bebidas alcoólicas no padrão binge em uma amostra clínica de usuários de cocaína (inalada e fumada) em tratamento para dependência química. Uma das grandes preocupações em relação a esse comportamento é que representa riscos diversos para a saúde do indivíduo, favorecendo condições para o surgimento de doenças sexualmente transmissíveis, hipertensão arterial severa, acidente vascular cerebral, doenças hepáticas e danos neurológicos ${ }^{(1-6)}$. Além da exposição dos usuários em situações de vulnerabilidade psicossocial que potencializam seu envolvimento em situações de violência (física entre parceiros íntimos, doméstica, brigas, acidentes de transporte) ${ }^{(1)}$.

A amostra foi composta por 140 (100\%) participantes, caracterizados predominantemente por serem adultos, do sexo masculino, solteiros, de cor negra/parda, com baixo nível de escolaridade e de religião católica. No entanto, não houve diferenças na amostra quando se comparam as características sociodemográficas e o consumo em binge (Tabela 1). As condições sociodemográficas e o contexto social do indivíduo são fatores importantes que interferem no planejamento da assistência e desfecho do tratamento $^{(17)}$. 
O uso de crack associado ao consumo de múltiplas drogas foi predominante entre os usuários entrevistados. Independentemente do uso dessas drogas, $113(80,7 \%)$ consumiram bebidas alcoólicas no padrão binge. Por ser uma amostra específica de indivíduos em tratamento, essas porcentagens podem ser consideradas elevadas. O beber em binge caracteriza-se como um comportamento muito comum, principalmente em homens jovens, pois o álcool é bastante aceito socialmente ${ }^{(1-2,18)}$.

A identificação do beber em binge, bem como suas consequências em usuários de cocaína e crack, tem sido fundamental para monitoração do padrão de consumo e planejamento de ações interventivas precoces diante dos problemas que esse comportamento pode ocasionar. Estudos recomendam envidar esforços para elucidar a relação entre as variáveis (cocaína versus binge), de forma a investigar as associações existentes entre o comportamento do beber e suas consequências agudas e crônicas ${ }^{(10,19)}$.

No presente estudo, os participantes que beberam em binge apresentaram frequência de consumo mais intensa, ou seja, uma a duas vezes por semana e diariamente, quando comparados àqueles que não consumiram nesse padrão, com diferenças estatisticamente significantes (Tabela 2). Conhecer o padrão de consumo de uma pessoa que bebe em níveis de risco torna-se cada vez mais necessário na prática do enfermeiro, para o reconhecimento dos riscos à saúde e aplicação de intervenções pontuais para minimizar os problemas decorrentes desse comportamento ${ }^{(17)}$.

O hábito do beber em binge mostrou-se diferenciado na amostra. Entre os usuários de múltiplas drogas, houve predomínio do uso regular nos últimos 30 dias e consumo diário, quando comparados aos usuários de cocaína e crack, com diferenças estatisticamente significantes (Tabela 3). A esse respeito, a literatura descreve que o álcool é a substância mais consumida por usuários de múltiplas drogas e, entre os bebedores pesados, há parcela considerável que faz uso de cocaína e $\operatorname{crack}^{(13)}$.

Estudo realizado com 111 pacientes, em sua maioria do sexo masculino, adultos, de cor negra, sem vínculo empregatício, que procuraram tratamento ambulatorial e foram diagnosticados com dependência de álcool e cocaína, identificou que os participantes preferiam usar substâncias combinadas ao uso isolado, o que pode ser explicado pela soma dos efeitos simultâneos, ou redução daqueles causados pelo uso de apenas uma droga ${ }^{(20)}$.

Quanto à síndrome da dependência do álcool, os usuários que consumiram no padrão binge foram classificados no nível de gravidade moderada (Tabela 4). Além disso, um resultado que chama a atenção é observado na comparação dos valores médios entre níveis de gravidade do uso de álcool, que foi maior entre os que beberam em binge.

Os resultados corroboram os de outras pesquisas que evidenciam que indivíduos que fizeram uso de álcool no padrão binge apresentaram maiores chances de desenvolver dependência do álcool. Conhecer os padrões de consumo e níveis de severidade da dependência do álcool e outras drogas permite traçar diretrizes para a implementação de ações e intervenções de saúde ${ }^{(21)}$.

Outro importante resultado observado no presente estudo refere-se às situações de violências. Os participantes que consumiam no padrão binge apresentaram maiores porcentagens de envolvimento em 
situações de violência (briga, violência física, comportamentos agressivos, encorajamento a lutar para se defender) (Tabela 4). A esse respeito, a literatura mostra que o beber em binge está potencialmente relacionado a riscos de morbimortalidade que podem ser prevenidos precocemente. Além disso, aumenta a probabilidade de envolvimento em situações de violências, seja na condição de agressor, seja na posição de vítima ${ }^{(22)}$. Há evidência de forte associação entre o beber em binge e o envolvimento em brigas e, nesse sentido, o consumo de álcool e drogas ilícitas é observado em diversos eventos violentos. Estudo com 1.631 indivíduos concluiu que o número de vítimas e agressores foi maior em situações que envolviam o consumo de álcool no padrão binge, totalizando $18,2 \%$ de vítimas e $15,9 \%$ de agressores ${ }^{(23)}$.

Há evidência de que até $92 \%$ dos episódios de violência notificados podem ser atribuídos aos efeitos do consumo de substâncias psicoativas ${ }^{(24)}$. Outro estudo, com 102 indivíduos usuários de cocaína inalada e fumada, revelou que $35 \%$ da população investigada referiram beber em binge pelo menos uma vez na semana. Identificou-se que, além dos indivíduos serem frequentes bebedores pesados, os usuários de crack envolvem-se mais em problemas e são mais propensos a usar drogas ilícitas, além do álcool ${ }^{(25)}$. As consequências desse uso, no entanto, foram ignoradas por muito tempo, e ainda são poucos os estudos brasileiros que relacionam o beber em binge e as situações de violência. Apesar de serem evidentes os riscos de danos decorrentes dessa combinação, os profissionais de saúde ainda são poucos sensibilizados e capacitados para enfrentar essa realidade.

Uma limitação do presente estudo refere-se ao fato de ter sido realizado em apenas um serviço especializado no tratamento da dependência química, o que faz com que a amostra não seja representativa da população de usuários de substâncias psicoativas. Assim, os dados devem ser apreciados com cautela quando comparados a outras amostras não oriundas de serviços especializados.

Os resultados obtidos reafirmam a necessidade de desenvolver novas investigações que abordem a temática com amostras mais robustas e a utilização de outras abordagens metodológicas, como estudos de seguimento, que possam explorar a estabilidade do padrão de consumo e sua progressão ao longo do tempo.

\section{CONCLUSÃO}

Este é um dos poucos estudos brasileiros que avaliam o beber em binge em uma amostra clínica de usuários de drogas em tratamento. Observou-se que o beber em binge é um hábito muito comum nessa população e parece associado a níveis severos de dependência tanto do álcool como de outras drogas. Este estudo também coloca em relevo o fato de que $80,7 \%$ dos usuários de drogas consumiram álcool no padrão binge, com destaque para os níveis severo e moderado de gravidade. Identificou-se a exposição à diversas situações de violência, no entanto, as associações com o consumo de álcool não se mostraram significantes. Esses dados têm impacto na prática profissional na medida em que oferece subsídios para o planejamento e sistematização das ações de enfermagem, a partir da utilização de instrumentos padronizados e culturalmente sensíveis aos segmentos mais vulneráveis da população.

O consumo de álcool é um problema de saúde pública complexo, que afeta inúmeras dimensões da 
vida em comunidade. O profissional de enfermagem desempenha papel fundamental na assistência aos usuários de álcool e outras drogas, considerando seu contato mais prolongado com esses pacientes nos serviços de saúde. Os achados trazem subsídios importantes sobre o beber em binge em usuários de drogas, o que auxilia os profissionais não somente a se capacitarem para o reconhecimento e identificação dos problemas inerentes ao beber pesado, mas também para a formulação de estratégias de intervenção motivacionais que auxiliam na mudança de comportamento, de forma a atender às necessidades integrais dessas pessoas. Essas ações, aplicadas em conjunto, podem contribuir para o aprimoramento da qualidade da assistência prestada, sem negligenciar a importância da oferta de práticas preventivas por meio da educação e promoção de saúde nos diversos serviços.

\section{Agradecimentos}

Agradecimentos ao CNPq. Auxílio Pesquisa Processo 482442/2011-5 e FAPESP: 2009/14861-2 pelos financiamentos concedidos para realização da presente pesquisa.

\section{REFERÊNCIAS}

1. World Health Organization. Global status report on alcohol and health [Internet]. Geneva: World Health Organization; 2011 [acesso em: 30 jun. 2016]. Disponível em: http://www.who.int/substance_abuse/publications/global_alcohol_report/msbgsruprofiles.pdf.

2. Laranjeira R, Madruga CS, Pinsky I, Caetano R, Mitsuhiro SS, Castello G. II Levantamento Nacional de Álcool e Drogas (LENAD) - 2012 [Internet] São Paulo: INPAD/UNESP; 2013[acesso em: 30 jun. 2016]. Disponível em:

http://inpad.org.br/wp-content/uploads/2014/03/Lenad-Il-Relatório.pdf.

3. Vargens RW, Cruz MS, Santos MA. Comparação entre usuários de crack e de outras drogas em serviço ambulatorial especializado de hospital universitário. Rev Lat Am Enfermagem [Internet]. 2011 [acesso em: 30 jun.

2016];19(spe):804-12. Disponível em: http://dx.doi.org/10.1590/S0104-11692011000700019.

4. Arantes LFR. Binge drinking: um estudo bibliométrico (1999-2010) dos artigos publicados na base de dados SciELO. Estud Psicol [Internet]. 2012 [acesso em: 30 jun. 2016];29(2):253-7. Disponível em: http://dx.doi.org/10.1590/S0103$166 \times 2012000200011$.

5. Nunes JM, Campolina LR, Vieira MA, Caldeira AP. Consumo de bebidas alcoólicas e prática do binge drinking entre acadêmicos da área da saúde. Rev Psiquiatr Clínica [Internet]. 2012 [acesso em: 30 jun. 2016];39(3):94-9. Disponível em: http://dx.doi.org/10.1590/S0101-60832012000300005.

6. Castroand DS, Sanchez ZM, Zaleski M, Alves HN, Pinsky I, Caetano R, et al. Sociodemographic characteristics associated with binge drinking among Brazilians. Drug Alcohol Depend [Internet]. 2012 [acesso em: 30 jun. 2016];126(1-2):272-6. Disponível em: http://dx.doi.org/10.1016/j.drugalcdep.2012.05.017.

7. Kanny D, Liu Y, Brewer RD. Vital signs: binge drinking prevalence, frequency, and intensity among adults - United States, 2010. Morb Mortal Wkly Rep [Internet]. 2012 [acesso em: 30 jun. 2016];61(1):14-9. Disponível em:

http://www.cdc.gov/mmwr/preview/mmwrhtml/mm6101a4.htm?s cid=mm6101a4_w.

8. Abdala N, Grau LE, Zhan W, Shaboltas AV, Skochilov RV, Kozlov AP, et al. Inebriation, drinking motivations and sexual risk taking among sexually transmitted disease clinic patients in St. Petersburg, Russia. AIDS Behav [Internet]. 2013 [acesso em: 30 jun. 2016];17(3):1144-50. Disponível em: http://dx.doi.org/10.1007/s10461-011-0091-z. 9. De Oliveira LG, Barroso LP, Silveira CM, Sanchez ZV, De Carvalho Ponce J, Vaz LJ, et al. Neuropsychological assessment of current and past crack cocaine users. Subst Use Misuse [Internet]. 2009 [acesso em: 30 jun. 2016];44(13):1941-57. Disponível em: http://dx.doi.org/10.3109/10826080902848897.

10. Ferri C, Marsden J, Araujo M, Laranjeira R, Gossop M. Validity and reliability of the Severity of Dependence Scale (SDS) in a Brazilian sample of drug users. Drug Alcohol Rev [Internet]. 2000 [acesso em: 30 jun. 2016];19(4):451-5. Disponível em: http://doi.wiley.com/10.1080/713659418. 
11. Scheffer M, Pasa GG, Almeida RMM. Dependência de álcool, cocaína e crack e transtornos psiquiátricos. Psicol Teor Pesqui [Internet]. 2010 [acesso em: 30 jun. 2016];26(3):533-41. Disponível em: http://dx.doi.org/10.1590/S010237722010000300016.

12. Miguel Ángel A-C, Laura B-S. Cocaetileno y Violencia: Influencia de la Interacción Cocaína-Alcohol en la Conducta Antisocial. Portada Anu Psicol Jurídica [Internet]. 2011 [acesso em: 30 jun. 2016];21:49-55. Disponível em: http://dx.doi.org/10.5093/jr2011v21a5.

13. Hedden SL, Malcolm RJ, Latimer WW. Differences between adult non-drug users versus alcohol, cocaine and concurrent alcohol and cocaine problem users. Addict Behav [Internet]. 2009 [acesso em: 30 jun. 2016];34(3):323-6. Disponível em: http://dx.doi.org/10.1016/j.addbeh.2008.11.001.

14. Rosa-Oliveira LQ, Presti PF, Antunes IR, Carbonari GC, Imada AC, Maeda MY, et al. Reliability and dimensionality of the Short Alcohol Dependence Data (SADD) questionnaire in a clinical sample of hospitalized patients: using the SADD in a general hospital setting. Rev Bras Psiquiatr [Internet]. 2011 [acesso em: 30 jun. 2016];33(1):68-71. Disponível em: http://dx.doi.org/10.1590/S1516-44462010005000020.

15. Bradley KA, DeBenedetti AF, Volk RJ, Williams EC, Frank D, Kivlahan DR. AUDIT-C as a Brief Screen for Alcohol Misuse in Primary Care. Alcohol Clin Exp Res [Internet]. 2007 [acesso em: 30 jun. 2016];31(7):1208-17. Disponível em: http://doi.wiley.com/10.1111/j.1530-0277.2007.00403.x.

16. Kaye S, Darke S. Determining a diagnostic cut-off on the Severity of Dependence Scale (SDS) for cocaine dependence. Addiction [Internet]. 2002 [acesso em: 30 jun. 2016];97(6):727-31. Disponível em:

http://doi.wiley.com/10.1046/j.1360-0443.2002.00121.x.

17. Souza LM, Pinto MG. Atuação do enfermeiro a usuários de álcool e de outras drogas na Saúde da Família. Rev. Eletr. Enf. [Internet]. 2012 [acesso em: 30 jun. 2016];14(2):374-83. Disponível em:

http://dx.doi.org/10.5216/ree.v14i2.11245.

18. Laranjeira R, Pinsky I, Zaleski M, Caetano R, Cuarte PCAV. I Levantamento Nacional sobre os padrões de consumo de álcool na população brasileira [Internet]. Brasília: Secretaria Nacional Antidrogas; 2007 [acesso em: 30 jun. 2016 ]. Disponível em: http://bvsms.saude.gov.br/bvs/publicacoes/relatorio_padroes consumo_alcool.pdf.

19. Elbreder MF, Silva RS, Pillon SC, Laranjeira R. Alcohol Dependence: Analysis of Factors Associated with Retention of Patients in Outpatient Treatment. Alcohol Alcohol [Internet]. 2011 [acesso em: 30 jun. 2016];46(1):74-6. Disponível em: http://dx.doi.org/10.1093/alcalc/agq078.

20. Horta RL, Horta BL, Rosset AP, Horta CL. Perfil dos usuários de crack que buscam atendimento em Centros de Atenção Psicossocial. Cad Saude Publica [Internet]. 2011 [acesso em: 30 jun. 2016];27(11):2263-70. Disponível em: http://dx.doi.org/10.1590/S0102-311X2011001100019.

21. Brites RMR, Abreu AMM. Padrão de consumo de bebidas alcoólicas entre os trabalhadores e perfil socioeconômico. Acta Paul Enferm [Internet]. 2014 [acesso em: 30 jun. 2016];27(2):93-9. Disponível em: http://dx.doi.org/10.1590/1982-0194201400018.

22. Zaleski M, Silva GL. Violência e uso, abuso e dependência de substâncias psicoativas. In: Diehl A, Cordeiro CC, Laranjeira R. Dependência química: prevenção, tratamento e políticas públicas. Porto Alegre: Artmed; 2011. p. $279-87$. 23. Oliveira JB, Lima MCP, Simão MO, Cavariani MB, Tucci AM, Kerr-Corrêa F. Violência entre parceiros íntimos e álcool: prevalência e fatores associados. Rev Panam Salud Pública [Internet]. 2009 [acesso em: 30 jun. 2016];26(6):494-501. Disponível em: http://www.scielosp.org/scielo.php?script=sci_arttext\&pid=S102049892009001200004\&lng=en\&nrm=iso\&tlng=pt.

24. Bes TM, Rozales Lopes FA, Morgan GJ, Ribeiro MS, Duarte WR. Relação da violência intrafamiliar e o uso abusivo de álcool ou entorpecentes na cidade de Pelotas, RS. Revista da AMRIGS [Internet]. 2013 [acesso em: 30 jun.

2016];57(1):9-13. Disponível em: http://www.amrigs.com.br/revista/57-01/1108.pdf.

25. Gossop M, Manning V, Ridge G. Concurrent use and order of use of cocaine and alcohol: behavioural differences between users of crack cocaine and cocaine powder. Addiction [Internet]. 2006 [acesso em: 30 jun.

2016];101(9):1292-8. Disponível em: http://doi.wiley.com/10.1111/j.1360-0443.2006.01497.x. 